\title{
Screening of kinase inhibitors targeting BRAF for regulating autophagy based on kinase pathways
}

\author{
YINGMEI ZHANG ${ }^{1}$, DONGBO XUE ${ }^{2}$, XIAOCHUN WANG ${ }^{2},{\text { MING } \mathrm{LU}^{3}, \mathrm{BO} \mathrm{GAO}^{2} \text { and XIN QIAO }}^{3}$ \\ ${ }^{1}$ Central Laboratory, The First Affiliated Hospital of Harbin Medical University, Harbin, Heilongjiang 150001; \\ ${ }^{2}$ Department of General Surgery, The First Affiliated Hospital of Harbin Medical University, Harbin, \\ Heilongjiang 150001, P.R. China; ${ }^{3}$ Department of Surgery, David Geffen School of Medicine, \\ University of California at Los Angeles, Los Angeles, CA 90064, USA
}

Received September 19, 2013; Accepted October 28, 2013

DOI: $10.3892 / \mathrm{mmr} .2013 .1781$

\begin{abstract}
The aim of this study was to identify agents that regulate autophagy. A total of 544 differentially expressed genes were screened from the intersection set of GSE2435 and GSE31040, which was obtained from the Gene Expression Omnibus database and 19 differentially expressed kinases were selected according to a 'protein kinase database'. Gene ontology-biological process (GO-BP) enrichment analysis revealed that the 19 kinases were mainly associated with phosphorylation. The protein-protein interaction network exhibited 30 differentially expressed genes that interacted with BRAF, and GO-BP enrichment analysis showed the function of these genes were mainly involved in cell death and apoptosis. The kinase-kinase inhibitor regulatory network identified16 kinase inhibitors that specifically inhibited BRAF. Previous studies indicated that sorafenib is capable of regulating autophagy and regorafenib has also been reported; however, there have been no studies regarding the regulation of autophagy by afatinib, selumetinib, PD318088, axitinib, TAK-733, GDC-0980, GSK2126458, PLX-4720, AS703026, trametinib, GDC-0941 and PF-04217903. Thus, these kinase inhibitors are potential targets for further study on the regulation of autophagy in the future.
\end{abstract}

\section{Introduction}

Autophagy refers to a series of biochemical processes in eukaryotic cells involving 'self-digestion' by degrading their own cytoplasm and organelles. Under nutritional deficiency, starvation or anoxia, autophagy is described as a pathway capable of promoting cell survival (1). Under these conditions, it

Correspondence to: Mrs Xiaochun Wang, Department of General Surgery, The First Affiliated Hospital of Harbin Medical University, No. 23 Youzheng Street, Nangang, Harbin, Heilongjiang 150001, P.R. China

E-mail: xiaochun_wang88@sina.com

Key words: autophagy, protein kinase, kinase inhibitor, bioinformatics is essential for autophagy to provide energy for cells. A previous study indicated that autophagy is associated with the effects of antitumor agents on tumor cells. Subsequently, tumor cells overcome nutritional deficiency and hypoxia by autophagy and are therefore protected from entering the apoptotic pathway (2).

One of the important factors for the poor prognosis of tumors is their tolerance to radiotherapy and chemotherapy. Recently, it was found that autophagy is one of the mechanisms involved in the resistance of tumors to chemotherapy (3). Autophagy inhibitor, chloroquine, improves the sensitivity of human lymphoma cells to apoptosis inducers, and thus it is considered to treat tumors by combining autophagy inhibitors and apoptosis inducers (4). Tumor cells grow in low-vascularized environments by autophagy and the treatment of tumors may be elucidated by inhibiting autophagic activities. Li et al (5) found that the inhibition of autophagy may improve the sensitivity of intestinal cancer cells to fluorouracil (5-FU). Therefore, it is of great importance to identify anti-tumor agents that target the molecular mechanisms of autophagy.

According to the concept of bioinformatics and network pharmacology, theoretically, if the inhibition of autophagic genes (or gene knockout) prevents autophagy, agents inhibiting the same genes may also suppress autophagy (6). At present, there are $\sim 500$ biological targets for therapeutic agents and it is estimated that human genome data may contain $~ 5,000-10,000$ novel targets, which is 10 - to 20 -fold higher than currently known targets. It is not difficult to obtain a number of novel agents, which may be 10-fold higher than the currently available drugs if genomic data are sufficiently mined (7). The present study screened differentially expressed protein kinases during autophagy using bioinformatics and constructed the kinase-kinase inhibitor regulatory network. The aim therefore was to explore kinase inhibitors capable of arresting autophagy with potential research values.

\section{Materials and methods}

Data resources. Data of gene expression chip analysis of starve-induced autophagy was obtained from the Gene Expression Omnibus (GEO) database at the intersection set of GSE2435 (http://www.ncbi.nlm.nih.gov/geo/query/acc. cgi?acc=GSE2345) and GSE31040 (http://www.ncbi.nlm.nih. 
Table I. Summary of the two data series from the GEO database.

\begin{tabular}{|c|c|c|c|c|c|}
\hline Series & Samples & Group & Experimental design & Array platform & References \\
\hline GSE2435 & $\begin{array}{l}\text { GSM45796 } \\
\text { GSM45797 } \\
\text { GSM45798 } \\
\text { GSM45799 } \\
\text { GSM45800 } \\
\text { GSM45801 }\end{array}$ & $\begin{array}{l}\text { Autophagy-6 h-1 } \\
\text { Autophagy-24 h-1 } \\
\text { Autophagy-24 h-2 } \\
\text { Control-6 h-1 } \\
\text { Control-24 h-1 } \\
\text { Control-24 h-2 }\end{array}$ & $\begin{array}{c}\text { Human } \\
\text { B-lymphoblastoid } \\
\text { cell line after } 6 \mathrm{~h} \\
\text { and } 24 \mathrm{~h} \text { of starvation }\end{array}$ & $\begin{array}{l}\text { GPL570 } \\
\text { HG-U133-Plus-2 } \\
\text { Affymetrix Human } \\
\text { Genome U133 } \\
\text { Plus 2.0 Array }\end{array}$ & $\begin{array}{l}\text { Proc Natl } \\
\text { Acad Sci } \\
\text { USA, } 2005 \\
\text { PMID: } \\
\text { 15894616 (8) }\end{array}$ \\
\hline GSE31040 & $\begin{array}{l}\text { GSM769043 } \\
\text { GSM769045 } \\
\text { GSM769049 } \\
\text { GSM769042 } \\
\text { GSM769044 } \\
\text { GSM769048 }\end{array}$ & $\begin{array}{l}\text { Autophagy-48 h-1 } \\
\text { Autophagy-48 h-2 } \\
\text { Autophagy-48 h-3 } \\
\text { Control-48 h-1 } \\
\text { Control-48 h-2 } \\
\text { Control-48 h-3 }\end{array}$ & $\begin{array}{c}\text { Human } \\
\text { lymphoblastoid } \\
\text { cell lines after } 48 \mathrm{~h} \\
\text { of starvation }\end{array}$ & $\begin{array}{l}\text { GPL570 } \\
\text { HG-U133-Plus-2 } \\
\text { Affymetrix Human } \\
\text { Genome U133 } \\
\text { Plus 2.0 Array }\end{array}$ & $\begin{array}{l}\text { Age (Dordr) } \\
\text { 2011 PMID: } \\
21904824 \text { (9) }\end{array}$ \\
\hline
\end{tabular}

gov/geo/query/acc.cgi?acc=GSE31040) as shown in Table I. The CEL data compression package of microarray was downloaded in the supplementary file of GEO and decompressed into another folder for further use. Moreover, the original data for the sample in TXT format was also downloaded.

Microarray data processing. mRNA microarray in the present study was performed as follows: Three samples from Homo sapiens were prepared under 'Control' and 'Autophagy' conditions, RMA algorithm was used to calculate the expression level and MAS algorithm was used to calculate the detection call. The probe numbers showing low expression levels were filtered using a P-value for a minimum of two detection calls to preserve any group of samples, and human HGNC gene abbreviation was used to unify the gene names. LIMMA differential gene screening algorithm was used to screen for the upregulated and downregulated genes under 'Autophagy' conditions relative to 'Control' conditions, and differential multiples, P-value and FDR values were calculated. $\mathrm{P}<0.05$ was considered to indicate differentially expressed genes.

The differentially expressed genes were screened by selecting the intersection set of GSE2435 and GSE31040, and the differentially expressed protein kinases were screened according to a 'protein kinase database' (http://kinasource. co.uk/Database /substrates.html).

Gene ontology (GO) enrichment analysis and cluster analysis on differentially expressed kinases. GO-BP (biological process) enrichment analysis was performed using DAVID software (10). Gene sets for further analysis were submitted to the DAVID database (http://david.abcc.ncifcrf.gov/), corresponding gene designators were simultaneously selected, human whole genome was ticked off as the background genes, the 'Functional Annotation Tool' was used as the analytical tool and the results for GO-BP enrichment analysis were obtained $(\mathrm{P}<0.05)$. Each GO-BP term and corresponding differentially expressed kinases were selected, and cytoscape software (version 2.6.3) was used to construct a visualized network to connect the kinases with their corresponding functions.
The differentially expressed protein kinases were identified to serve as the candidates for hierarchical cluster analysis using Cluster2.2 and TreeView3.2 software (11). The ratios of the coexpressed candidate kinases were transformed into $\log _{2} \%$, the distance was measured by means of Pearson correlation and cluster analysis was performed with single linkage clustering.

Construction of the kinase-gene interaction network. The protein-protein interaction (PPI) network was obtained from the HPRD database (http://www.hprd.org/), with 36,874 lines and 9,453 nodes being included in the network. The differentially expressed genes for analysis were projected into the PPI network and the correlation pairs in which the two interacting genes were differentially expressed were reserved, thus the sub-network was obtained. The differentially expressed kinase genes for further analysis were projected into the sub-network, the correlation pairs in which the differentially expressed kinases and the differentially expressed genes were interacting were reserved and thus the kinase-gene interaction network was obtained.

Screening of kinase inhibitors that regulate autophagy. An Excel file of 194 kinase inhibitors was established based on a database (http://www.selleckbio.com/servlet/Down loadServlet?fileName=Selleck-Kinase-Inhibitor-Library.xlsx). In the literature mining approach, Perl language software (www.perl.com) was used to write a program of literature mining. The Excel files of 'differentially expressed kinases' and 'kinase inhibitors' were imported to the ActivePerl 5.16.2 software, and the literature information was obtained from the National Library of Medicine's PubMed database (http://www. ncbi.nlm.nih.gov/pubmed/). The scope of the retrieval included titles and abstracts of all the articles in the PubMed database database. Valuable articles were identified by manual screening and we constructed the regulatory network by Cytoscape (version 2.6.3).

The name of each screened kinase inhibitor and 'Autophagy' were input into Pubmed for searching and the kinase inhibitors were divided into 3 groups $(0,1-10$ and $>11)$ according to the number of retrieved references. 
Table II. Differentially expressed protein kinase of starve-induced autophagic cells.

\begin{tabular}{|c|c|c|c|c|c|}
\hline \multirow[b]{2}{*}{ Gene symbol } & \multirow[b]{2}{*}{ Gene ID } & \multirow[b]{2}{*}{ Gene name } & \multicolumn{2}{|c|}{ P-value } & \multirow[b]{2}{*}{ Regulation } \\
\hline & & & GSE2435 & GSE31040 & \\
\hline CLK4 & 826442 & CDC-like kinase 4 & 0.006853 & 0.016876591 & Up \\
\hline NEK9 & 821660 & $\begin{array}{l}\text { NIMA (never in mitosis } \\
\text { gene a)-related kinase } 9\end{array}$ & 0.047605 & 0.018157559 & Down \\
\hline TLK2 & 809721 & Tousled-like kinase 2 & 0.010894 & 0.007442178 & Up \\
\hline RIOK3 & 815805 & RIO kinase 3 (yeast) & 0.016673 & 0.028184311 & Up \\
\hline TBCK & 811953 & $\begin{array}{l}\text { TBC domain-containing } \\
\text { protein kinase-like }\end{array}$ & 0.01651 & 0.007265638 & Down \\
\hline BRD2 & 773220 & Bromodomain containing 2 & 0.010431 & 0.004012674 & Up \\
\hline PRKCH & 774932 & Protein kinase $\mathrm{C}$, eta & 0.005525 & 0.008714029 & Down \\
\hline CSNK1A1 & 791771 & Casein kinase $1 \alpha 1$ & 0.007338 & 0.042396721 & Up \\
\hline IRAK4 & 807982 & $\begin{array}{l}\text { Interleukin-1 } \\
\text { receptor-associated kinase } 4\end{array}$ & 0.024849 & 0.029144612 & Down \\
\hline HIPK1 & 787073 & $\begin{array}{l}\text { Homeodomain interacting } \\
\text { protein kinase } 1\end{array}$ & 0.005074 & 0.005330583 & $\mathrm{Up}$ \\
\hline TRIB3 & 822826 & $\begin{array}{l}\text { Tribbles homolog } 3 \\
\text { (Drosophila) }\end{array}$ & 0.010471 & $1.33 \mathrm{E}-05$ & $\mathrm{Up}$ \\
\hline BRD4 & 799732 & Bromodomain containing 4 & 0.040358 & 0.012603665 & $\mathrm{Up}$ \\
\hline EIF2AK2 & 824980 & $\begin{array}{l}\text { Eukaryotic translation } \\
\text { initiation factor } 2-\alpha \\
\text { kinase } 2\end{array}$ & 0.002633 & 0.00920921 & Down \\
\hline CDK2 & 803206 & Cyclin-dependent kinase 2 & 0.030362 & 0.011442217 & Down \\
\hline BRAF & 821298 & $\begin{array}{l}\text { V-raf murine sarcoma viral } \\
\text { oncogene homolog B1 }\end{array}$ & 0.01214 & 0.016176288 & Up \\
\hline PASK & 811281 & $\begin{array}{l}\text { PAS domain containing } \\
\text { serine/threonine kinase }\end{array}$ & 0.003506 & 0.00239591 & Down \\
\hline CLK1 & 823347 & CDC-like kinase 1 & 0.000355 & 0.000302227 & Up \\
\hline TXK & 810008 & $\begin{array}{l}\text { TXK tyrosine kinase } \\
\text { v-akt murine thymoma }\end{array}$ & 0.00019 & 0.001451441 & $\mathrm{Up}$ \\
\hline AKT1 & 791692 & Viral oncogene homolog 1 & 0.033324 & 0.011637129 & Down \\
\hline
\end{tabular}

\section{Results}

Differentially expressed kinases and their function. In total 13,234 genes were detected in GSE2435 and 2,783 differentially expressed genes were screened. A total of 14,442 genes were detected in GSE31040 and 2,369 differentially expressed genes were screened. A total of 544 genes were allocated in the intersection set for the differentially expressed genes screened out from GSE2435 and GSE31040.

Among the 544 differentially expressed genes, 19 genes encoding protein kinases were screened out (as shown in Table II), of which 11 genes were upregulated and 8 genes were downregulated. The 11 upregulated genes were used as targets for further intervention by kinase inhibitors.

Results of the GO-BP enrichment analysis (Fig. 1) revealed 19 kinases were mainly associated with functions, such as protein amino acid phosphorylation (GO:0006468), phosphorylation (GO:0016310), phosphorus metabolic process (GO:0006793), phosphate metabolic process (GO:0006796), protein amino acid autophosphorylation (GO:0046777, intracellular signaling cascade (GO:0007242), protein kinase cascade (GO:0007243) and peptidyl-serine phosphorylation (GO:0018212).

A visualized network was constructed to exhibit the correlation between kinases and GO-BP terms, results showed all kinases were associated with 'phosphorus metabolic process', 'phosphorylation' and 'protein amino acid phosphorylation', which indicated that these kinases were important in the process of phosphorylation. In addition, BRAF, IRAK, TXK and AKT1 were also associated with 'protein kinase cascade' and BRAF, IRAK, TXK, AKT1, HIPK1, PRKCH and TLK2 with intracellular signaling cascade; suggesting that these kinases should be further studied (Fig. 2).

Cluster analysis (Fig. 3) of these 19 kinases indicated that the expression of 11 kinases were upregulated (BRD4, TRIB3, BRD2, TLK2, HIPK1, CLK4, RIOK3, BRAF, TXK, CSNK1A1 and CLK1) and these should be potential targets for further study. Among them, BRAF, was closely associated with RIOK3, and HIPK1 with CLK4, therefore regulation of one kinase may induce synchronic changes of its correlated kinase. 


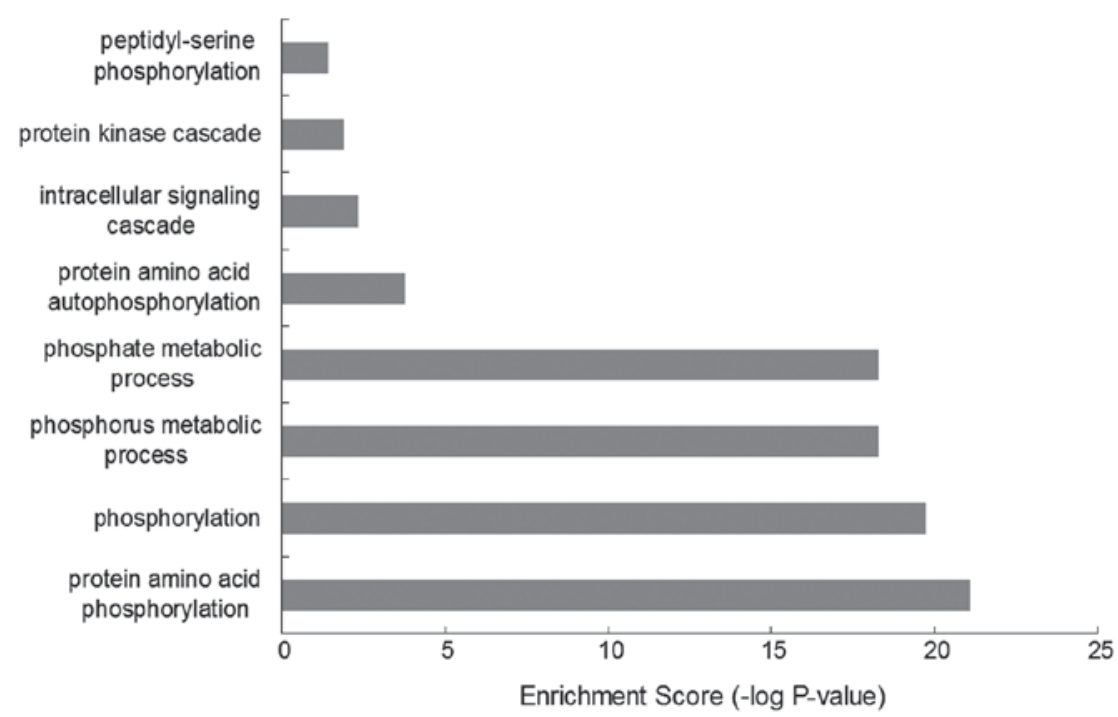

Figure 1. GO-BP enrichment analysis of the 19 differentially expressed protein kinases.

Role and function of BRAF in regulating autophagy. As shown in Fig. 4, the network constructed based on the PPI network showed that BRAF interacted with the differentially expressed genes, such as CASP3, FAS, GRB2, HLA-A, HLA-G, HRAS, ICAM1, IFNAR1, IFNAR2, KRAS, MAP2K1, MAP2K2, MAPK1, NFATC1, NFATC4, NRAS, PIK3CA, PIK3CB, PIK3CD, PIK3R1, PLCG1, PRF1, PRKCA, PRKCB, PTPN11, RAC1, RAF1, SOS1, TNF and TNFRSF10D. The enrichment analysis of these differentially expressed genes annotated the function of BRAF; however, it may be presumed that the inhibition of BRAF may have caused the synchronized alteration of these genes.

GO-BP enrichment analysis showed that the function of these BRAF-associated genes were mainly involved in biological processes, such as 'apoptosis' (GO:0006915), 'programmed cell death' (GO:0012501), 'regulation of apoptosis' (GO:0042981), 'regulation of programmed cell death' (GO:0043067), 'regulation of cell death' (GO:0010941), 'cell death' (GO:0008219), 'induction of apoptosis by extracellular signals' (GO:0008624), 'negative regulation of apoptosis (GO:0043066), 'negative regulation of programmed cell death' (GO:0043069), 'negative regulation of cell death' (GO:0060548), 'induction of apoptosis' (GO:0006917), 'induction of programmed cell death' (GO:0012502), 'positive regulation of apoptosis' (GO:0043065), 'positive regulation of programmed cell death' (GO:0043065) and 'positive regulation of cell death' (GO:0010942), which infer that the regulation of BRAF may effect the mechanisms of cell death (Figs. 5 and 6).

Screening result of BRAF-targeting kinase inhibitors. It was found by constructing the kinase-kinase inhibitor regulatory network (Fig. 7) that SB, PP-121, Torisel, PD98059, imatinib, CI-1040, roscovitine, everolimus, dasatinib and lapatinib simultaneously regulated BRAF, AKT4 and CDK2, while AKT4 and CDK2 were downregulated; thus these kinase inhibitors were excluded. Vandetanib and PD0325901 simultaneously inhibited BRAF and CDK2, therefore these two kinase inhibitors were also excluded. Crizotinib, pelitinib, PI-103, BEZ235, sunitinib and AZD8055 simultaneously inhibited BRAF and
AKT1, thus these 6 kinase inhibitors were also excluded. The remaining 16 kinase inhibitors specifically suppressed BRAF, among which 39 references and 2 references reported that sorafenib and regorafenib regulated autophagy, respectively, while there was no report for GDC-0879, RAF265, afatinib, selumetinib, PD318088, axitinib, TAK-733, GDC-0980, GSK2126458, PLX-4720, AS703026, trametinib, GDC-0941 or PF-04217903 of regulating autophagy. This finding indicated that these kinase inhibitors are potential targets for further investigation on the regulations of autophagy.

\section{Discussion}

A number of anti-tumor treatments may induce autophagy (12). It was found that in breast cancer cells, tamoxifen and other estrogen receptor antagonists induced autophagy (13). Chemotherapy at varied doses may result in autophagy at different degrees in breast cancer cells, colorectal cancer cells and prostate cancer cells (14). Autophagy of tumor cells may be induced during treatment on spongiocytoma using arsenic trioxide, temozolomide and rapamycin (15), treatments on ovarian cancer using resveratrol (16) and treatments on cervical cancer using histone deacetylase (17). Therefore, it has been estimated that the therapeutic alliance of autophagic inhibitors may improve therapeutic efficacy and prognosis (18). Mathew et al (19) demonstrated from animal testing that the inhibition of autophagy may aid anti-tumor therapy. They indicated that a large concentration of tumor cells died following cytotoxic treatment, while autophagy promoted resilience and hibernation of tumor cells, and autophagy inhibitors may directly eliminate these cells. When the autophagy inhibitor, chloroquine, was used in combination with alkylating agents, satisfactory therapeutic efficacy was obtained (20). The phenomenon of the induction of apoptosis by autophagy inhibitors was observed in previously mentioned studies (21); therefore, sensitization may be induced in anti-tumor treatments if autophagy inhibitors were used in combination to block the autophagic pathway. However, only a limited number of autophagy inhibitors are 


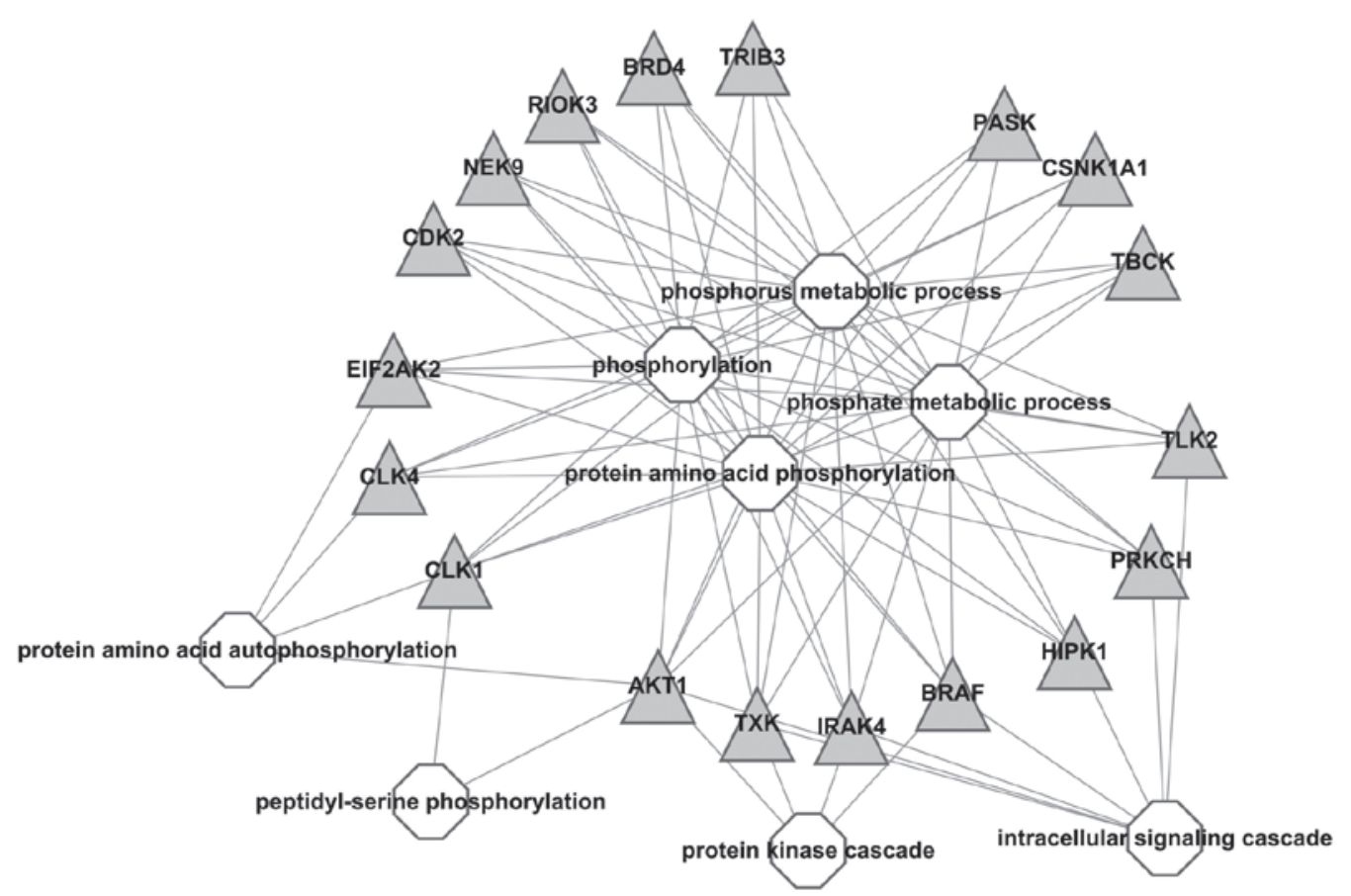

Figure 2. Network of GO-BP term and their corresponding protein kinases. The triangular-shaped nodes refer to protein kinases and the octagon-shaped nodes refer to GO-BP terms.

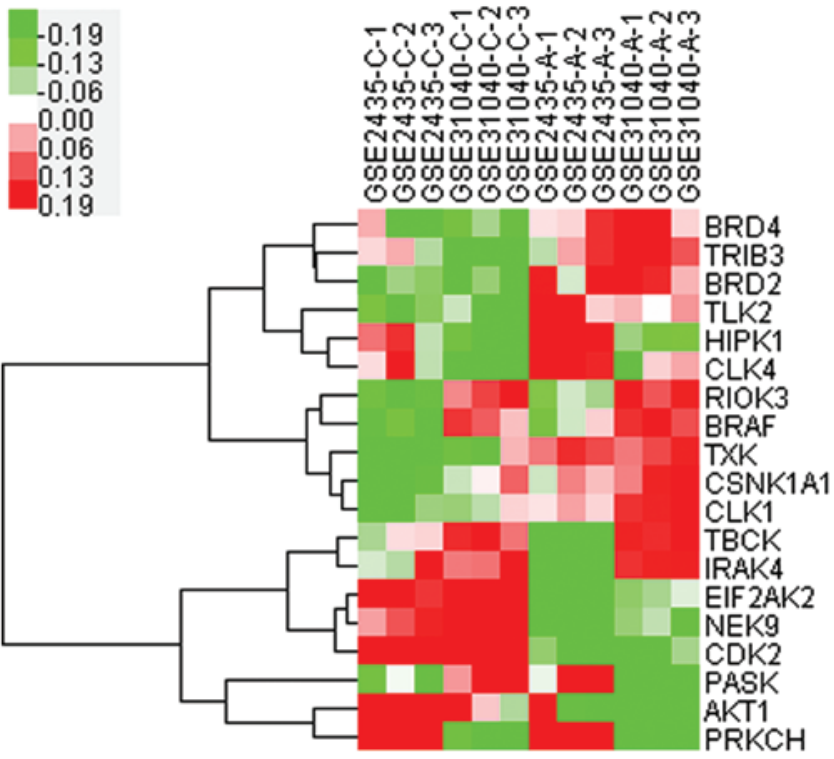

Figure 3. Cluster analysis of the differentially expressed protein kinases.

available for current clinical application, including bafilomycin and chloroquine (22).

Numerous molecules are involved in autophagy and the regulatory mechanisms are extremely complex. The majority of regulatory mechanisms remain unclear; however, a number of efforts have been made for exploration and many signaling molecules are shared in regulating autophagy and apoptosis. The determined signaling molecules involved in regulating autophagy include cytokine, MAPK and mitochondrial signaling for which most of the signals exert regulatory functions by mTOR kinase (23).
Molecular switches accurately control proteins during cascade reactions for a series of signaling transduction processes in cells by activation or deactivation mechanisms. Among the cascade reactions for a series of signaling transduction processes, positive and negative feedback mechanisms complementing each other are essential for accurate control, thus the function of molecule switches is influential (24). The proteins involved in signaling transduction processes include protein kinases and GTP-binding proteins. Protein kinases control the activities of $30 \%$ of the proteins in cells, which are associated with almost all of the cell functions (25). Protein kinases are particularly important for intracellular signaling transduction and implementation of complex cell functions, such as cell division and death. The core function of kinases in controlling cell behaviors subsequently caused them to be considered as targets for treating multiple diseases and thus they have been extensively investigated. In addition, in contrast to gene transfection and RNA interference, a number of small molecular compounds are capable of effectively regulating kinase activities (26). They have been commercialized and it is of practical significance to introduce them to switch autophagy, which may realize the transformation of laboratory achievements to clinical application, and meets with the concepts of translational medicine. Therefore, the present study examined the factors regulating autophagy. In total, 544 differentially expressed genes were screened using the genome-wide expression profile microarray in 2 groups of autophagy models induced by starvation, among which 11 upregulated protein kinases were used as the targets for intervention by kinase inhibitors. Among the upregulated kinases, BRAF and TRIB3 were found to have corresponding specific inhibitors in the kinase-kinase inhibitor regulatory network. Moreover, numerous inhibitors were available for BRAF (27) and thus BRAF was analyzed. 


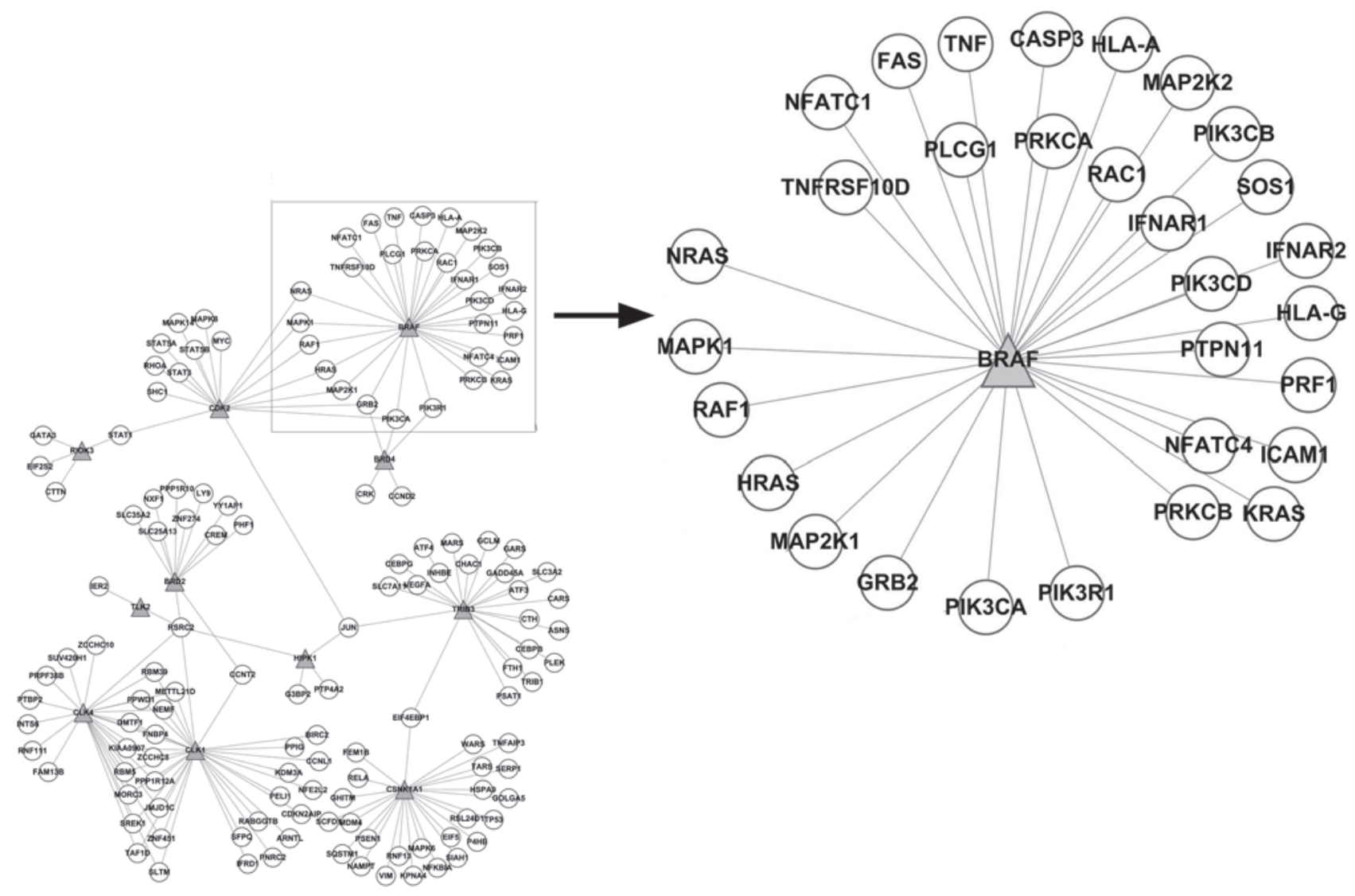

Figure 4. Network of the differentially expressed protein kinases (left) and the network of BRAF and its interacting genes (right). The triangular-shaped nodes refer to protein kinases and the elliptical-shaped nodes refer to differentially expressed genes.

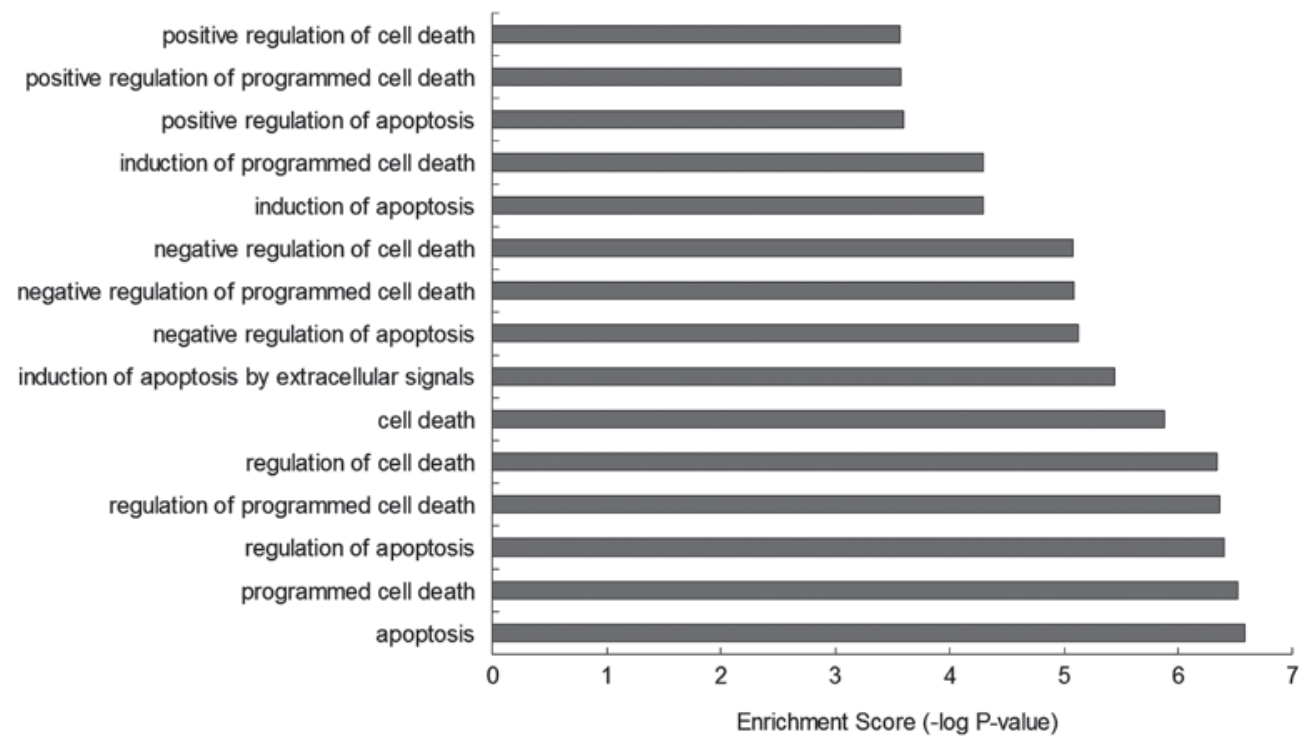

Figure 5. GO-BP enrichment analysis of the differentially expressed genes that interacted with BRAF.

$\mathrm{BRAF}$ is one of the three isoforms of RAF and it is an important transduction factor in the MAPK pathway (28). The MAPK signaling pathway is one of the most important signaling pathways in cells, it exists in most cells and transmits extracellular stimuli into cells or even nuclei. It has important functions in inducing proliferation, differentiation, transforma- tion, apoptosis and other biological reactions (29). Extracellular signals stimulate ERK and RAS, and RAS further activates RAF and phosphorylates MAPK kinase (MEK1/MEK2). ERKs are translocated into the nucleus, phosphorylated cytosolic proteins and certain nuclear transcription factors, such as c-FOS, c-JUN, ELK-1, c-MYC and ATF2; therefore, they are 


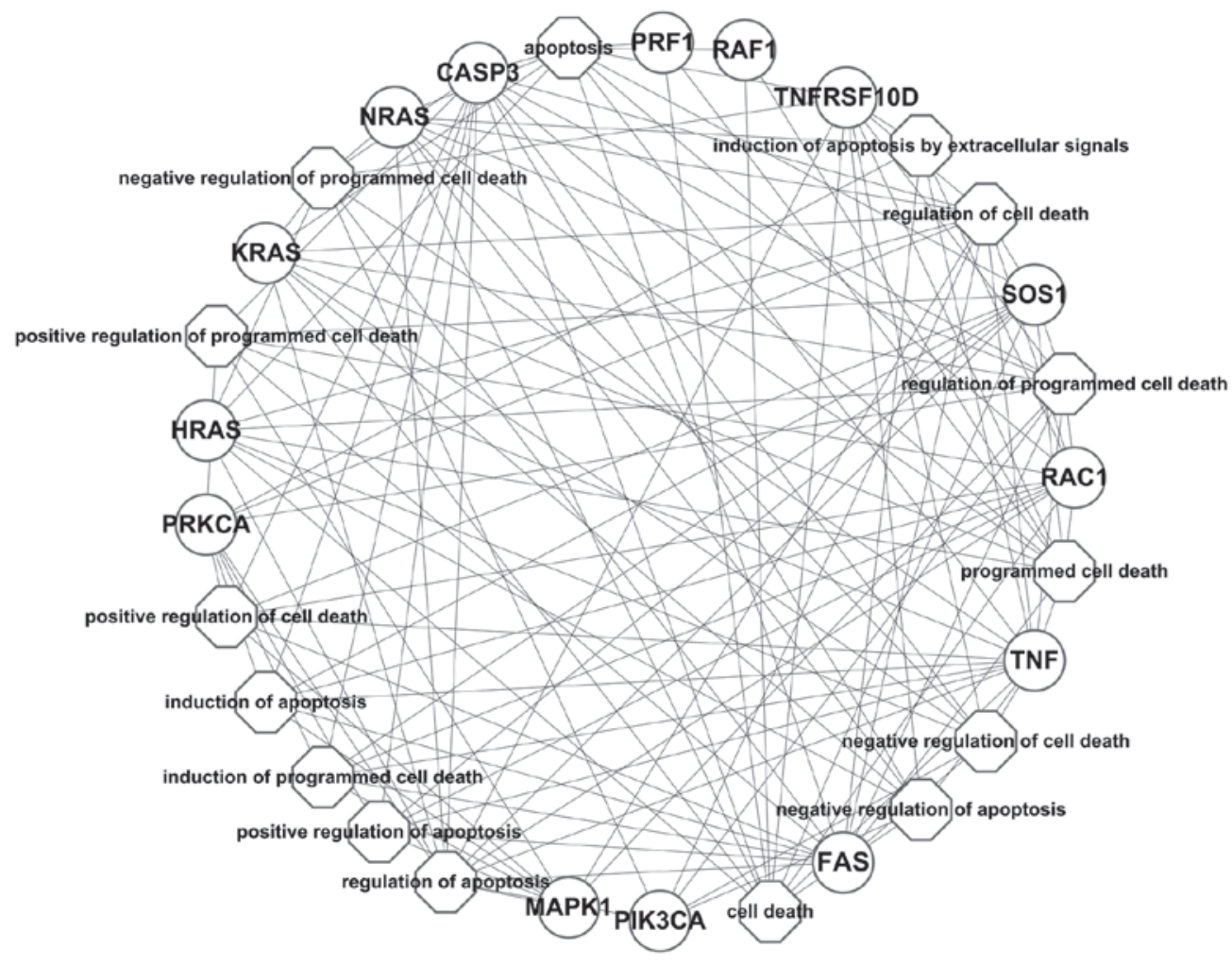

Figure 6. Network of GO-BP term and their corresponding BRAF interacting genes. The elliptical-shaped nodes refer to BRAF interacted differentially expressed genes, and the octagon-shaped nodes refer to GO-BP terms.

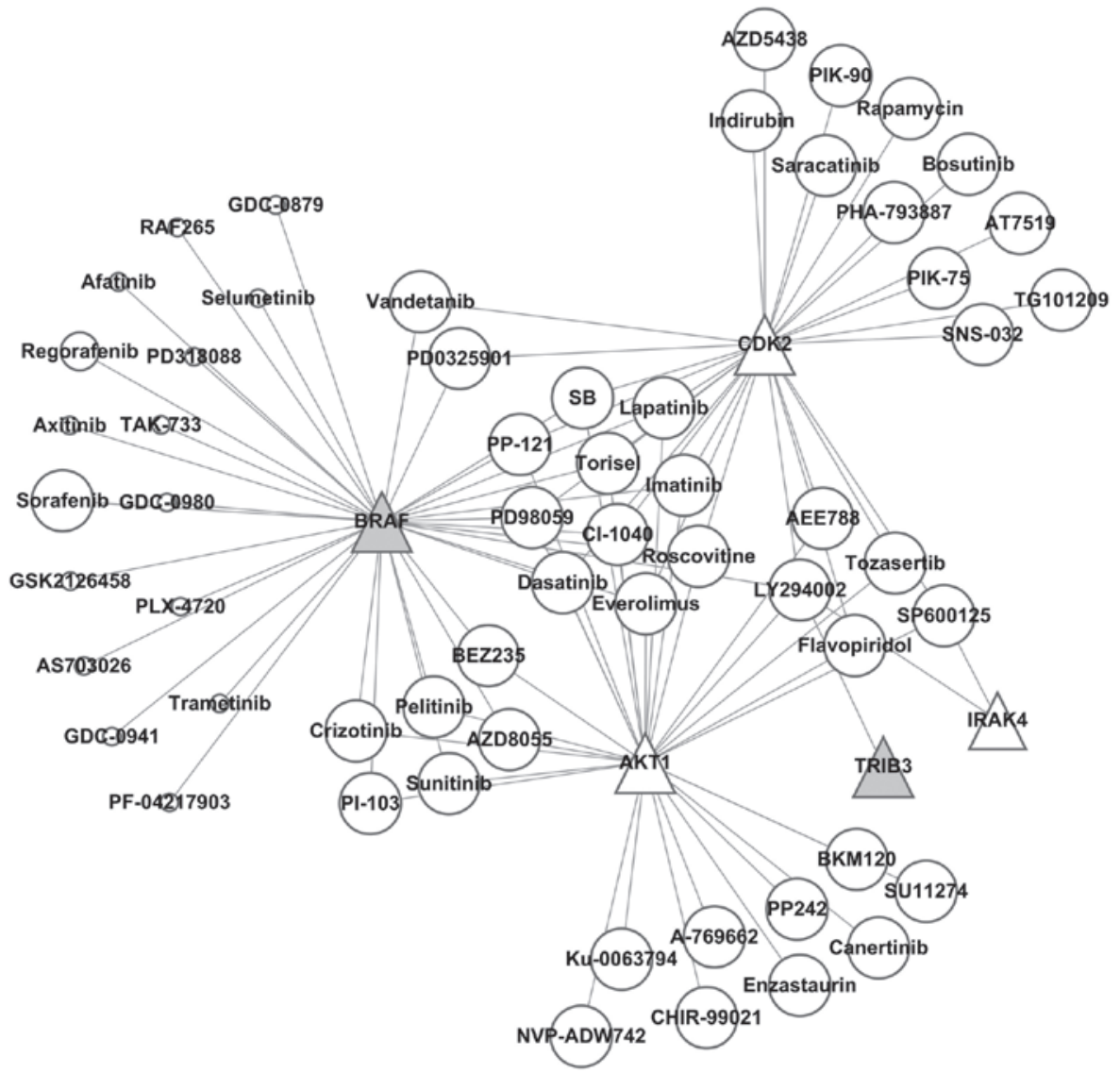

Figure 7. Network of kinase inhibitors and their corresponding protein kinases. Each triangular-shaped node refers to protein kinases, and the elliptical-shaped nodes refer to kinase inhibitors. The large elliptical-shaped node means $>11$ reports regarding the regulation of autophagy by this kinase inhibitor were identified, the middle one refers to 1-10 reports, the small one refers to no reports in PubMed. 
involved in the regulation of cell proliferation and differentiation. ERKl/2 is activated by the MAPK pathway by survival signals and promotes autophagy by PI3K via mTOR (30). Shimada et al (31) indicated that endoplasmic reticulum stress may induce autophagy by activating the MAPK signaling pathway. To determine the functions of BRAF, the present study constructed a correlation network between BRAF and the differentially expressed genes regulated by BRAF. GO-BP enrichment analysis on these differentially expressed genes showed that their major functions were associated with cell death and apoptosis; therefore, regulation of BRAF may result in significant effects on the mechanisms of cell death. Results of the present study have shown that RIOK 3 was closely associated with BRAF by cluster analysis. RIOK3 was very important for maintaining normal physiological function of cells, and RIOK3 depletion may result in cell death or significant abnormality in physiological function. Shan et al (32) reported that RIOK3 may be involved in the regulation of apoptosis by coordinating caspase-10, caspase- 2 and NF- $\mathrm{KB}$ activation, indicating that RIOK3 may be closely associated with cell death.

The present study has demonstrated by constructing the kinase-kinase inhibitor regulatory network, that certain kinase inhibitors had multiple targets, and may inhibit downregulated genes (AKT4 and CDK2) and simultaneously inhibit upregulated genes (BRAF), thus off-target side-effects may occur and should be excluded. Of the 16 included specific kinase inhibitors functioning on BRAF, sorafenib was previously confirmed to be capable of regulating autophagy, while regorafenib had also been reported. However, no report was available on the regulation of autophagy by afatinib, selumetinib, PD318088, axitinib, TAK-733, GDC-0980, GSK2126458, PLX-4720, AS703026, trametinib, GDC-0941 and PF-04217903. Thus, these kinase inhibitors are potential targets for investigating the regulation of autophagy in the future.

\section{Acknowledgements}

This study was supported by the National Natural Science Foundation of China (grant no. 81370566).

\section{References}

1. Efeyan A, Zoncu R, Chang S, et al: Regulation of mTORC1 by the Rag GTPases is necessary for neonatal autophagy and survival. Nature 493: 679-683, 2013.

2. Wang ZB, Peng XZ, Chen SS, et al: High p53 and MAP1 light chain $3 \mathrm{~A}$ co-expression predicts poor prognosis in patients with esophageal squamous cell carcinoma. Mol Med Rep 8: 41-46, 2013.

3. Yao F, Wang G, Wei W, Tu Y, Tong H and Sun S: An autophagy inhibitor enhances the inhibition of cell proliferation induced by a proteasome inhibitor in MCF-7 cells. Mol Med Rep 5: 84-88, 2012.

4. Amaravadi RK, Yu D, Lum JJ, et al: Autophagy inhibition enhances therapy-induced apoptosis in a Myc-induced model of lymphoma. J Clin Invest 117: 326-336, 2007.

5. Li J, Hou N, Faried A, Tsutsumi S, Takeuchi T and Kuwano H: Inhibition of autophagy by 3-MA enhances the effect of 5-FU-induced apoptosis in colon cancer cells. Ann Surg Oncol 16: 761-771, 2009.

6. Yildirim MA, Goh KI, Cusick ME, Barabási AL and Vidal M: Drug-target network. Nat Biotechnol 25: 1119-1126, 2007.

7. Hopkins AL: Network pharmacology: the next paradigm in drug discovery. Nat Chem Biol 4: 682-690, 2008.

8. Dengjel J, Schoor O, Fischer R, et al: Autophagy promotes MHC class II presentation of peptides from intracellular source proteins. Proc Natl Acad Sci USA 102: 7922-7927, 2005.
9. Matarrese P, Tinari A, Ascione B, et al: Survival features of EBV-stabilized cells from centenarians: morpho-functional and transcriptomic analyses. Age (Dordr) 34: 1341-1359, 2012.

10. Hong MG, Pawitan Y, Magnusson PK and Prince JA: Strategies and issues in the detection of pathway enrichment in genome-wide association studies. Hum Genet 126: 289-301, 2009.

11. Juan HF and Huang HC: Bioinformatics: microarray data clustering and functional classification. Methods Mol Biol 382: 405-416, 2007.

12. Wu T, Li Y, Gong L, et al: Multi-step process of human breast carcinogenesis: a role for BRCA1, BECN1, CCND1, PTEN and UVRAG. Mol Med Rep 5: 305-312, 2012.

13. Scarlatti F, Bauvy C, Ventruti A, et al: Ceramide-mediated macroautophagy involves inhibition of protein kinase B and up-regulation of beclin 1. J Biol Chem 279: 18384-18391, 2004.

14. Paglin S, Hollister T, Delohery T, et al: A novel response of cancer cells to radiation involves autophagy and formation of acidic vesicles. Cancer Res 61: 439-444, 2001.

15. Kanzawa T, Zhang L, Xiao L, Germano IM, Kondo Y and Kondo S: Arsenic trioxide induces autophagic cell death in malignant glioma cells by upregulation of mitochondrial cell death protein BNIP3. Oncogene 24: 980-991, 2005.

16. Opipari AW Jr, Tan L, Boitano AE, Sorenson DR, Aurora A and Liu JR: Resveratrol-induced autophagocytosis in ovarian cancer cells. Cancer Res 64: 696-703, 2004.

17. Takeuchi H, Kondo Y, Fujiwara K, et al: Synergistic augmentation of rapamycin-induced autophagy in malignant glioma cells by phosphatidylinositol 3-kinase/protein kinase B inhibitors. Cancer Res 65: 3336-3346, 2005.

18. Jin S and White E: Role of autophagy in cancer: management of metabolic stress. Autophagy 3: 28-31, 2007.

19. Mathew R, Karantza-Wadsworth V and White E: Role of autophagy in cancer. Nat Rev Cancer 7: 961-967, 2007.

20. Carew JS, Nawrocki ST, Kahue CN, et al: Targeting autophagy augments the anticancer activity of the histone deacetylase inhibitor SAHA to overcome Bcr-Abl-mediated drug resistance. Blood 110: 313-322, 2007.

21. Hashimoto K and Sakagami H: Induction of apoptosis by epigallocatechin gallate and autophagy inhibitors in a mouse macrophage-like cell line. Anticancer Res 28: 1713-1718, 2008.

22. Amaravadi RK and Thompson CB: The roles of therapy-induced autophagy and necrosis in cancer treatment. Clin Cancer Res 13: 7271-7279, 2007.

23. Nazio F, Strappazzon F, Antonioli M, et al: mTOR inhibits autophagy by controlling ULK1 ubiquitylation, self-association and function through AMBRA1 and TRAF6. Nat Cell Biol 15: 406-416, 2013.

24. Wang ZJ and Wang LX: Phosphorylation: a molecular switch in opioid tolerance. Life Sci 79: 1681-1691, 2006.

25. Pelkmans L, Fava E, Grabner H, et al: Genome-wide analysis of human kinases in clathrin- and caveolae/raft-mediated endocytosis. Nature 436: 78-86, 2005.

26. Bellodi C, Lidonnici MR, Hamilton A, et al: Targeting autophagy potentiates tyrosine kinase inhibitor-induced cell death in Philadelphia chromosome-positive cells, including primary CML stem cells. J Clin Invest 119: 1109-1123, 2009.

27. Liu D, Liu Z, Jiang D, Dackiw AP and Xing M: Inhibitory effects of the mitogen-activated protein kinase kinase inhibitor CI-1040 on the proliferation and tumor growth of thyroid cancer cells with BRAF or RAS mutations. J Clin Endocrinol Metab 92: 4686-4695, 2007.

28. Derdas SP, Soulitzis N, Balis V, Sakorafas GH and Spandidos DA: Expression analysis of B-Raf oncogene in V600E-negative benign and malignant tumors of the thyroid gland: correlation with late disease onset. Med Oncol 30: 336, 2013.

29. Xu Y, Zhang J and Dong WG: Indole-3-carbinol (I3C)-induced apoptosis in nasopharyngeal cancer cells through Fas/FasL and MAPK pathway. Med Oncol 28: 1343-1348, 2011.

30. Thorburn A: Apoptosis and autophagy: regulatory connections between two supposedly different processes. Apoptosis 13: 1-9, 2008.

31. Shimada Y, Kobayashi H, Kawagoe S, et al: Endoplasmic reticulum stress induces autophagy through activation of p38 MAPK in fibroblasts from Pompe disease patients carrying c.546G>T mutation. Mol Genet Metab 104: 566-573, 2011.

32. Shan J, Wang P, Zhou J, Wu D, Shi $\mathrm{H}$ and Huo K: RIOK3 interacts with caspase-10 and negatively regulates the NF-kappaB signaling pathway. Mol Cell Biochem 332: 113-120, 2009. 